\title{
INVARIANT ARCS, WHITNEY LEVELS, AND KELLEY CONTINUA
}

\author{
M. VAN DE VEL
}

\begin{abstract}
As an application of convexity in spaces of arcs, three results of a somewhat different nature have been obtained. The first one gives some simple conditions under which an arc of a semilattice is mapped back into itself by an order-preserving function. The second result states that certain Whitney levels are absolute retracts. Finally, Kelley continua are characterized by what we call approximating coselections.
\end{abstract}

\section{INTRODUCTION}

Let $S$ be a partially ordered space (a pospace). By an arc in $S$ is meant a totally ordered subcontinuum. Throughout, $\Gamma(S)$ denotes the collection of all arcs in $S$, and $\Lambda(S)$ denotes the subcollection of all arcs joining a minimal and a maximal element of $S$. In most situations, $S$ will be a connected Lawson (join) semilattice. In particular, $S$ is compact and hence it has a top element 1. As a consequence of Koch's Arc Theorem, each point of $S$ is linked to 1 by an arc; cf. [G\&, VI.5.11].

Spaces of arcs in hyperspaces of metric continua have been considered in [C, ENN], and (to some extent) in [V2]. For a geometric application involving arcs in cubes, see [V3].

Theorem I. Let $S$ be a connected Lawson semilattice with a bottom element $\mathbf{0}$, and let $f: S \rightarrow S$ be a continuous order-preserving function, such that $f(\mathbf{0})=\mathbf{0}$. Then there is an arc $A \in \Lambda(S)$ with $f(A) \subseteq A$.

We will actually prove a more general coincidence theorem below.

Theorem II. Let $S$ be an arcwise connected metrizable Lawson semilattice with a bottom element $\mathbf{0}$, such that the order intervals $[x, y]$ for $x \leq y$ in $S$ depend continuously on their endpoints. If $X \subseteq S$ is a closed set of incomparable points, separating between $\mathbf{0}, \mathbf{1}$, then $X$ is an absolute retract.

Even for $S$ a metric cube, this result seems to be new. For a different example, let $w: S \rightarrow[0,1]$ be a Whitney map (cf. [W] or $\S 4$ below) and

Received by the editors November 30, 1984 and, in revised form, July 22, 1989.

1980 Mathematics Subject Classification (1985 Revision). Primary 54H12, 52A01, 54B20; Secondary $54 \mathrm{H} 25,54 \mathrm{C} 65,54 \mathrm{~F} 40$.

Key words and phrases. Absolute retract, approximating coselection, arc, continuous selection, convex set, Kelley continuum, Lawson semilattice, Whitney map. 
consider the level set $w^{-1}(t)$, where $0<t<1$. For related results, see [P, $\mathrm{GN}]$.

Let $Z$ be a metric continuum. Then $H(Z)$ denotes its hyperspace of compacta and $C(Z)$ denotes the subspace of all continua. The space $Z$ is said to be a Kelley Continuum (or, to have property $K$; cf. [N, p. 538]) provided for each $\varepsilon>0$ there exists $\delta>0$ with the following property. If $a, b \in Z$ and $A \in C(Z)$ are such that $d(a, b)<\delta$ and $a \in A$, then there exists $B \in C(Z)$ with $b \in B$ and $d(A, B)<\varepsilon$ (Hausdorff distance). Examples are: Peano continua, hereditarily indecomposable continua, and homogeneous continua.

The third main result of this paper is on Kelley continua and involves the following concept. A continuous compact-valued function $F: Z \rightarrow H(Z)$ with $z \in F(z)$ for all $z \in Z$ is called a coselection, [N, p. 265]. We say that $F$ is $\varepsilon$-approximating (where $\varepsilon>0$ ) provided each $F(z)$ is a continuum and for all $a \in A \in C(Z)$ there exists $z \in Z$ with

$$
d(a, z)<\varepsilon, \quad d(A, F(z))<\varepsilon .
$$

Roughly speaking: $F$ approximates the "membership relation."

Theorem III. Let $Z$ be a Kelley continuum. Then for each $\varepsilon>0$ there exists an $\varepsilon$-approximating coselection.

In fact, given $C_{0} \in C(Z)$ and $c_{0} \in C_{0}$ there even exists an $\varepsilon$-approximating coselection $F$ with $F\left(c_{0}\right)=C_{0}$. This property characterizes Kelley continua.

All proofs involve a convex structure on the space of all order arcs. Here, convexity is based on a natural betweenness concept for arcs and it has been inspired by a recent paper of Curtis [C], where a notion of "convex combination" has been developed for arcs in hyperspaces. Our concept fits in with a theory of "abstract" convexity as developed in [ $\mathrm{J} 1, \mathrm{~V} 1]$, and the results obtained in [V2] apply. Theorem I follows from a fixed point theorem for a class of convex structures. In Theorem II, retractions are constructed with the aid of a selection theorem, and finally, a result on approximating convex-valued functions by single-valued ones, yields Theorem III.

There are several reasons for operating with Lawson semilattices instead of the more restricted class of hyperspaces. Throughout, members of hyperspaces (compacta, continua) are treated as "points", the individual structure of which is irrelevant at most places. Moreover, in the proof of the third theorem, we consider a space of type $H(Z) \times[0,1]$, which is a semilattice but not a hyperspace. To capture the essence of Kelley continua in these circumstances, we have introduced the concept of a Kelley semilattice.

The organization is as follows. Some preliminary concepts, notation, and results are collected in $\S 1$. Spaces of order arcs in semilattices with a top element are studied in combination with "abstract" convexity. This is done in $\S 2$, where we verify the main conditions required in [V2] for an application of the results developed there. For semilattices with more than one minimal element, some extra complications arise which we handle in $\S 3$. The main theorems- 
Theorems I, II, III, are derived and commented upon in $\S 4$.

Some of the results of $\S 2$ have been announced in (an earlier manuscript of) [V2].

\section{Preliminaries}

1.1. Hyperspaces and Lawson semilattices. Let $Z$ be a compact Hausdorff space. If $A_{1}, \ldots, A_{n} \subseteq Z$ then $\left\langle A_{1}, \ldots, A_{n}\right\rangle$ denotes the family $\{A \mid A \subseteq$ $\left.\bigcup_{i=1}^{n} A_{i} ; \forall i=1, \ldots, n: A \cap A_{i} \neq \varnothing\right\}$. The hyperspace $H(Z)$ of $Z$ consists of all nonempty closed subsets, equipped with the Vietoris topology. A base of open sets in this topology is formed by the sets of type $\left\langle O_{1}, \ldots, O_{n}\right\rangle$, where $n \in \mathbb{N}$ and $O_{i} \subseteq Z$ is open for each $i$. See [M, N]. The space $H(Z)$ can be partially ordered by inclusion, yielding a semilattice with $A \cup B$ as the supremum (join) of $A, B$.

By a Lawson semilattice is meant a (join) semilattice with a compact topology making the join operation continuous, and that each such point has a neighborhood base consisting of subsemilattices. For instance, the hyperspace of a compact Hausdorff space is a Lawson semilattice (the basic open sets described above are subsemilattices). For a general semilattice we use $x \vee y$ to denote the supremum (join) and

$$
\begin{aligned}
\leq(x) & =\{y \mid y \leq x\} \\
{[x, y] } & =\{z \mid x \leq z \leq y\} \quad(x \leq y) .
\end{aligned}
$$

1.2. Uniformity, closeness. The canonical uniformity of a compact space $Z$ is denoted by $\mu(Z)$. It is conceived as a collection of symmetric entourages of the diagonal in $Z \times Z$; cf. [E, p. 523]. For $V \in \mu(Z)$, we abbreviate $V \circ V$ as $2 V$. The following notions of closeness are standard. Two points $a, b \in Z$ are $V$-close provided $(a, b) \in V$ and two $n$-tuples $\left(a_{i}\right)_{i=1}^{n},\left(b_{i}\right)_{i=1}^{n}$ in $Z$ are $V$-close provided $a_{i}, b_{i}$ are $V$-close for each $i$. Two sets $A, B$ are $V$-close if each $a \in A$ is $V$-close to some $b \in B$ and vice versa. Finally, two families of sets $\mathscr{A}, \mathscr{B}$ are $V$-close if each $A \in \mathscr{A}$ is $V$-close to some $B \in \mathscr{B}$ and vice versa.

We note that these derived notions of closeness correspond with "induced" uniformities on, respectively, $Z^{n}, H(Z), H(H(Z))$.

1.3. Straightening. Let $S$ be a semilattice and let $\left(a_{1}, \ldots, a_{n}\right)$ be an $n$-tuple in $S$. Its straightening is defined to be the $n$-tuple $\left(b_{1}, \ldots, b_{n}\right)$, with $b_{i}=$ $\mathrm{V}_{j \leq i} a_{j}$. In the next two results, $S$ denotes a Lawson semilattice.

1.4. Lemma. For each $U \in \mu(S)$ there exists $V \in \mu(Z)$ such that for all $n \in \mathbb{N}$ the straightening of two $V$-close $n$-tuples are $U$-close.

Proof. $S$ being compact, each nonempty subset has a supremum; in a Lawson semilattice, the corresponding operation sup: $H(S) \rightarrow S$ is continuous, [G\&, p. 289]. Given $U \in \mu(S)$, choose $V \in \mu(S)$ such that the suprema of two $V$-close sets are $U$-close. 
1.5. Lemma. For each $U \in \mu(S)$ there exists $V \in \mu(Z)$ such that the following is true. If $A, B \subseteq S$ are chains and if

$$
\begin{aligned}
& a_{0}=\inf (A) \leq a_{1} \leq \cdots \leq a_{n}=\sup (A), \\
& b_{0}=\inf (B) \leq b_{1} \leq \cdots \leq b_{n}=\sup (B)
\end{aligned}
$$

are subsets of $A, B$ respectively, such that $a_{i}, b_{i}$ are $V$-close and $b_{i}, b_{i+1}$ are $V$-close for all $i$, then $A, B$ are $U$-close.

Proof. Consider the following refining sequence in $\mu(S)$ :

$$
V \subseteq 2 V \subseteq V_{2} \subseteq V_{1} \subseteq 2 V_{1} \subseteq U .
$$

In particular, the entourage $V_{2}$ is chosen such that for each $x \in S$ the orderconvex hull of $V_{2}(x)$ is included in $V_{1}(x)$ (local order-convexity; cf. [G\&, p. 273]).

If $b \in B$, say: $b_{i} \leq b \leq b_{i+1}$, then $b_{i}, b_{i+1} \in V\left(b_{i}\right) \subseteq V_{2}\left(b_{i}\right)$ and hence $b \in V_{1}\left(b_{i}\right)$. As $b_{i} \in V_{1}\left(a_{2}\right)$, we find that $b \in U\left(a_{i}\right)$.

Conversely, let $a \in A$, say: $a_{i} \leq a \leq a_{i+1}$. We have $a_{i} \in V\left(b_{i}\right)$ and $a_{i+1} \in$ $2 V\left(b_{i}\right)$. Hence $a_{i}, a_{i+1} \in V_{2}\left(b_{i}\right)$ and it follows that $a \in V_{1}\left(b_{i}\right) \subseteq U\left(b_{i}\right)$.

\section{SPACES OF ORDER ARCS; CONVEXITY}

Throughout this section, $S$ denotes a Lawson (join) semilattice with a bottom element $\mathbf{0}$. Note that a top element 1 exists by compactness. By [G\&, p. 299], the space $\Lambda(S)$ of all arcs joining $\mathbf{0}, \mathbf{1}$ is compact (it is empty if $S$ is disconnected).

2.1. Convex sets in $\Lambda(S)$. Let $A, A_{1}, \ldots, A_{n} \in \Lambda(S)$. We say that $A$ is in between $A_{1}, \ldots, A_{n}$ provided

$$
A \subseteq \bigvee_{i=1}^{n} A_{i}=\left\{a_{1} \vee \cdots \vee a_{n} \mid \forall i: a_{i} \in A_{i}\right\}
$$

A set $C \subseteq \Lambda(S)$ is said to be convex provided with each finite set of arcs, $C$ also contains any arc in between them. It is clear that the empty set, each singleton, and the entire set $\Lambda(S)$ are convex; that intersection of convex sets is convex, and that the union of an updirected collection of convex sets is convex.

An essential difference with convexity in vector spaces is the absense of "convex combinations" (see [C], however). Some terminology of "abstract" convexity will be of help:

2.2. Convexity. A convexity on a set $X$ is a collection $\mathscr{C}$ of subsets, to be thought of as the convex sets, among which are $\varnothing$ and $X$, and such that $\mathscr{C}$ is stable under intersections and updirected unions. If $A \subseteq X$ then its convex hull is the set

$$
\operatorname{co}(A)=\bigcap\{C \mid A \subseteq C \in \mathscr{C}\} .
$$

One of the axioms ensures that this is indeed a convex set. For $F$ finite, the set $\operatorname{co}(F)$ is called a polytope. The last axiom of convexity implies that a set $C$ 
is convex iff $F \subseteq C$ implies $\operatorname{co}(F) \subseteq C$. The pair $(X, \mathscr{C})$ is called a convex structure. We usually omit explicit reference to the convexity $\mathscr{C}$.

As for convexity in $\Lambda(S)$, it is clear that if the arcs $B_{1}, \ldots, B_{m}$ are in between the arcs $A_{1}, \ldots, A_{n}$, and if the arc $B$ is in between $B_{1}, \ldots, B_{m}$, then $B$ is in between $A_{1}, \ldots, A_{n}$-the transitive law of betweenness. Therefore, $\operatorname{co}\left\{A_{1}, \ldots, A_{n}\right\}$ consists exactly of all arcs in between the given ones.

A second example, needed later on, is the following one. Let $X$ be an arbitrary semilattice and let $a, a_{1}, \ldots, a_{n} \in X$. This time, we say that $a$ is in between $a_{1}, \ldots, a_{n}$ provided $a_{i} \leq a \leq \bigvee_{j=1}^{n} a_{j}$ for some $i$. The transitive law of betweenness applies here as well-mutatis mutandis-and we obtain a convexity on $X$ in which $\operatorname{co}\left\{a_{1}, \ldots, a_{n}\right\}$ consists exactly of all points which are in between the given ones. It can be verified that the convex sets are precisely the "order-convex" subsemilattices.

A half-space of a convex structure is a convex set with a convex complement. The separation axiom $S_{4}$ (also known as the Kakutani separation property) states that for each pair of disjoint convex sets $C, D$ there is a half-space $H$ with $C \subseteq H$ and $D \cap H=\varnothing$. It is a standard fact that vector spaces have this property with regard to the usual convexity. A semilattice with the convexity described above also enjoys this property, cf. [V2]. To establish the same fact for spaces of arcs requires some preparatory work.

A convex structure is called join-hull commutative provided for each point $x$ and for each nonempty convex set $C$ it is true that

$$
\operatorname{co}(\{x\} \cup C)=\bigcup_{c \in C} \operatorname{co}(x, c\} .
$$

Note that the inclusion from right to left is always valid; by the last axiom of convexity, it suffices to have the opposite inclusion for polytopes only.

\subsection{Proposition. For a join-hull commutative convex structure $X$ the following} assertions are equivalent.

(1) $X$ has the separation property $S_{4}$.

(2) If $a, a_{1}, a_{2} \in X$ and $b_{1} \in \operatorname{co}\left\{a, a_{1}\right\}, b_{2} \in \operatorname{co}\left\{a, a_{2}\right\}$, then

$$
\operatorname{co}\left\{a_{1}, b_{2}\right\} \cap \operatorname{co}\left\{a_{2}, b_{1}\right\} \neq \varnothing \text {. }
$$

Proof. Suppose first that $\operatorname{co}\left\{a_{1}, b_{2}\right\}$ and $\operatorname{co}\left\{a_{2}, b_{1}\right\}$ are disjoint and separate them with a half-space $H$ :

$$
\operatorname{co}\left\{a_{1}, b_{2}\right\} \subseteq H \quad \text { and } \quad \operatorname{co}\left\{a_{2}, b_{1}\right\} \subseteq X \backslash H .
$$

Then $a \in H$ yields $b_{1} \in \operatorname{co}\left\{a, a_{1}\right\} \subseteq H$ and $a \in X \backslash H$ yields $b_{2} \in \operatorname{co}\left\{a, a_{2}\right\} \subseteq$ $X \backslash H$, both a contradiction.

As for the converse, let $C, D \subseteq X$ be disjoint convex sets. By the last axiom of convexity there exist convex sets $C_{0} \supseteq C$ and $D_{0} \supseteq D$, maximal with the property $C_{0} \cap D_{0}=\varnothing$. Suppose there exists $a \in X \backslash\left(C_{0} \cup D_{0}\right)$. Then $\operatorname{co}\left(\{a\} \cup C_{0}\right)$ meets $D_{0}$ in a point $d_{1}$ by maximality, whereas by join-hull commutativity there is a point $c_{1} \in C_{0}$ with $d_{1} \in \operatorname{co}\left\{a, c_{1}\right\}$. We similarly obtain $c_{2} \in C_{0}$ 
and $d_{2} \in D_{0}$ such that $c_{2} \in \operatorname{co}\left\{a, d_{2}\right\}$. Now the sets $\operatorname{co}\left\{c_{1}, c_{2}\right\} \subseteq C_{0}$ and $\operatorname{co}\left\{d_{1}, d_{2}\right\} \subseteq D_{0}$ are disjoint; apparently, condition (2) fails.

2.4. Lemma. The convex structure $\Lambda(S)$ is join-hull commutative.

Proof. We consider some arcs as described below:

$$
A, B_{1}, \ldots, B_{n} \in \Lambda(S) ; \quad B \in \operatorname{co}\left\{A, B_{1}, \ldots, B_{n}\right\} .
$$

Then $B \subseteq \bigvee_{i} B_{i} \bigvee A$ and hence each $b \in B$ can be written in the form

$$
b=b_{1} \vee \cdots \vee b_{n} \vee a,
$$

where $b_{i} \in B_{i}$ and $a \in A$. By choosing each $b_{i}$ maximal in $B_{i}$ with the property $b \geq b_{i}$ we fix a function ()$_{i}: B \rightarrow B_{i}$. Clearly, $b \leq b^{\prime}$ in $B$ implies $b_{i} \leq b_{i}^{\prime}$ in $B_{i}$, and hence the set

$$
C^{\prime}=\left\{b_{1} \vee \cdots \vee b_{n} \mid b \in B\right\}
$$

is a chain. Now $C^{\prime} \subseteq \bigvee_{i} B_{i}$ and by the continuity of the join operator, we see that $\bigvee_{i} B_{i}$ is arcwise connected. Hence any extension of $C^{\prime}$ to a maximal chain in $\bigvee_{i} B_{i}$ must be an arc $C$ joining 0 with 1 . Then $C$ is in between the $\operatorname{arcs} B_{i}(i=1, \ldots, n)$ and $B$ is in between $C$ and $A$, establishing the result.

This allows us to derive the first main result.

\subsection{Theorem. $\Lambda(S)$ has the Kakutani separation property, $\mathbf{S}_{4}$.}

Proof. We work for an application of Proposition 2.3. Let $A, A_{1}, A_{2} \in \Lambda(S)$, and let $B_{i} \in \operatorname{co}\left\{A, A_{i}\right\}$ for $i=1,2$. We have a continuous semilattice homomorphism

$$
f: A_{1} \times A_{2} \times A \rightarrow S
$$

with $f\left(a_{1}, a_{2}, a\right)=a_{1} \vee a_{2} \vee a$. Let

$$
B_{1}^{\prime}=f^{-1}\left(B_{1}\right) \cap\left(A_{1} \times\{\mathbf{1}\} \times A\right),
$$

and let $f_{1}: B_{1}^{\prime} \rightarrow B_{1}$ be the restriction of $f$. Note that $f_{1}$ is surjective since $B_{1} \in \operatorname{co}\left\{A, A_{1}\right\}$. For each $s \in S$ the fiber

$$
f_{1}^{-1}(s)=f^{-1}(s) \cap\left(A_{1} \times\{\mathbf{1}\} \times A\right)
$$

is an order-convex subsemilattice of the square $A_{1} \times\{\mathbf{1}\} \times A$ and hence is connected. Now $f_{1}$ is a closed mapping onto a continuum and it has connected fibers. Hence its domain $B_{1}^{\prime}$ must be connected. Also, $B_{1}^{\prime}$ is a compact semilattice and $(\mathbf{0}, \mathbf{1}, \mathbf{0}),(\mathbf{1}, \mathbf{1}, \mathbf{1})$ are its bottom and top elements, respectively. By Koch's Arc Theorem there is an arc $C_{1} \subseteq B_{1}^{\prime}$ joining these two points.

Similarly, we obtain an arc $C_{2}$ in

$$
f^{-1}\left(B_{2}\right) \cap\left(\{1\} \times A_{2} \times A\right)
$$

joining $(\mathbf{1}, \mathbf{0}, \mathbf{0})$ and $(\mathbf{1}, \mathbf{1}, \mathbf{1})$. Consider the compact subsemilattice

$$
T=\left\{\left(a_{1}, a_{2}, a\right) \mid\left(a_{1}, \mathbf{1}, a\right) \in C_{1},\left(\mathbf{1}, a_{2}, a\right) \in C_{2}\right\} .
$$


Then $T$ contains $(\mathbf{0}, \mathbf{0}, \mathbf{0})$ and $(\mathbf{1}, \mathbf{1}, \mathbf{1})$, and we have a closed projection $T \rightarrow A$ onto the third axis. Each fiber of it is connected. Indeed, the fiber at $a \in A$ is either a rectangle (if both $C_{1}$ and $C_{2}$ are horizontal around $a$ ), or a line segment (if one of $C_{1}, C_{2}$ is horizontal around $a$ ), or a point (if none of $C_{1}, C_{2}$ are horizontal around $a$ ). As before, we conclude that $T$ is connected. By Koch's Arc Theorem again, we obtain an arc $C \subseteq T$ joining $(\mathbf{0}, \mathbf{0}, \mathbf{0})$ to $(\mathbf{1}, \mathbf{1}, \mathbf{1})$. Then $f(C)$ is an arc in $S$ connecting $\mathbf{0}$ and $\mathbf{1}$, and we verify that

$$
f(C) \in \operatorname{co}\left\{A_{2}, B_{1}\right\} \cap \operatorname{co}\left\{A_{1}, B_{2}\right\} .
$$

Take $s \in f(C)$, say: $s=f\left(a_{1}, a_{2}, a\right)$, where

$$
\left(a_{1}, \mathbf{1}, a\right) \in C_{1}, \quad\left(\mathbf{1}, a_{2}, a\right) \in C_{2} .
$$

Now $C_{1} \subseteq f^{-1}\left(B_{1}\right)$ whence $a_{1} \vee a \in B_{1}$ and

$$
s=\left(a_{1} \vee a\right) \vee a_{2} \in B_{1} \vee A_{2} .
$$

Similarly, $s \in B_{2} \vee A_{1}$, showing that $f(C)$ is in between $B_{1}, A_{2}$ and in between $B_{2}, A_{1}$.

We next concentrate on topological properties.

2.6. Theorem. All convex subsets of $\Lambda(S)$ are connected.

Proof. It suffices to show that for each two arcs $A_{0}, A_{1} \in \Lambda(S)$ the set $\operatorname{co}\left\{A_{0}, A_{1}\right\}$ includes a connected set containing $A_{0}, A_{1}$. To this end, consider the function

$$
f: A_{1} \rightarrow \Lambda(S), \quad f(t)=\left\{a_{1} \in A_{1} \mid a_{1} \leq t\right\} \cup\left\{t \vee a_{0} \mid a_{0} \in A_{0}\right\} .
$$

Clearly, each $f(t)$ is an arc in between $A_{0}$ and $A_{1}$, and $f$ is continuous. Moreover, $f(\mathbf{0})=A_{0}$ and $f(\mathbf{1})=A_{1}$.

2.7. Uniform Convexity. Let the set $X$ be equipped with a uniformity $\mu$ and with a convexity $\mathscr{C}$. The triple $(X, \mu, \mathscr{C})$ is called a uniform convex structure [V2], provided:

(i) All polytopes of $X$ are closed in the uniform topology of $(X, \mu)$.

(ii) For each $U \in \mu$ there exists $V \in \mu$ such that $V$-close sets have $U$-close hulls.

We are particularly interested in situations where $\mu$ is induced by a metric $d$. In these circumstances, the triple $(X, d, \mathscr{C})$ is called a metric convex structure.

2.8. Theorem. The convex structure $\Lambda(S)$ is uniform, and metric if $S$ is a metric space.

Proof. Recall that $\Lambda(S)$ is a compact subspace of $H(S)$, the hyperspace of $S$. The topology of $\Lambda(S)$ is then generated by a unique uniformity, namely, the one based on entourages of type

$$
\{(A, B) \mid A, B \in \Lambda(S) \text { are } V \text {-close }\} \quad(V \in \mu(S)) .
$$


Note that this is a metric uniformity if $\mu(S)$ is. A polytope $\operatorname{co}\left\{A_{1}, \ldots, A_{n}\right\}$ in $\Lambda(S)$ can be seen as the intersection of $\Lambda(S)$ with the closed subset

$$
\left\langle\bigvee_{i=1}^{n} A_{i}\right\rangle=\left\{A \in H(S) \mid A \subseteq \bigvee_{i=1}^{n} A_{i}\right\}
$$

of the hyperspace. This yields a compact set, which is uniformly closed.

We concentrate on the second requirement. Let $U \in \mu(S)$ and consider the following refining sequence of uniform entourages:

$$
V \subseteq V_{2} \subseteq V_{1} \subseteq U
$$

where

(1) $V_{1} \subseteq U$ is chosen as in Lemma 1.5;

(2) $V_{2} \subseteq V_{1}$ is chosen as in Lemma 1.4; and

(3) $V$-close sets in $S$ have $V_{2}$-close suprema.

Let $A_{1}, \ldots, A_{m}$ and $B_{1}, \ldots, B_{n}$ be $V$-close sets of arcs in $\Lambda(S)$. We verify that their convex hulls are $U$-close. To this end, take $A \in \operatorname{co}\left\{A_{1}, \ldots, A_{m}\right\}$ and fix an increasing sequence

$$
s_{0}=\mathbf{0} \leq s_{1} \leq \cdots \leq s_{k}=\mathbf{1}
$$

in $A$ in which successive points are $V$-close. Fix $l \in\{0, \ldots, k\}$ for a moment. The point $s_{l}$ can be represented as

$$
s_{l}=a_{l, 1} \vee \cdots \vee a_{l, m} \quad\left(a_{l, i} \in A_{i}\right) .
$$

Choose points $b_{l, j} \in B_{j}$ for $j=1, \ldots, n$ in such a way that the sets

$$
\left\{a_{l, i} \mid i=1, \ldots, m\right\} \text { and }\left\{b_{l, j} \mid j=1, \ldots, n\right\}
$$

are $V$-close. By (3), $s_{l}$ is $V_{2}$-close to the point

$$
t_{l}^{\prime}=b_{l, 1} \vee \cdots \vee b_{l, n} \in \bigvee_{j=1}^{n} B_{j}
$$

We may assume that $t_{0}^{\prime}=\mathbf{0}$ and that $t_{1}^{\prime}=\mathbf{1}$. By (2) and Lemma 1.4, the straightening $\left(t_{l}\right)_{l=0}^{k}$ of $\left(t_{l}^{\prime}\right)_{l=0}^{k}$ is $V_{1}$-close to $\left(s_{l}\right)_{l=0}^{k}$. Note that $t_{l}$, being a supremum of points in $\bigvee_{j=1}^{n} B_{j}$, is again in this (sub)semilattice. We extend the chain of all points $t_{l}$ to a maximal chain $B \subseteq \bigvee_{j=1}^{n} B_{j}$. The latter being an arcwise connected semilattice, we find that $B$ is an arc joining $\mathbf{0}, \mathbf{1}$. By construction, we have $B \in \operatorname{co}\left\{B_{1}, \ldots, B_{n}\right\}$. Finally, by (1) and Lemma 1.5, $B$ is $U$-close to $A$.

Reversing the role of the arcs $A_{i}$ and $B_{j}$, we conclude that the resulting polytopes are $U$-close, as announced.

Other examples of uniform or metric convex structures have been given in [V2]. For instance, a topological vector space (with its canonical translationinvariant uniformity) is a uniform convex structure iff it is locally convex. A 
compact Hausdorff semilattice $X$ (convexified as in 2.2) is a uniform convex structure iff it is locally convex (equivalently: is a Lawson semilattice) in which the order intervals $[x, y], x \leq y$, depend continuously on their endpoints. Its convex sets are all connected iff $X$ is arc-wise connected.

The results of this section allow for an application of the theory, developed in [V2]. For concepts involving multifunctions, we refer to [M]. Let us quote the main results to be used below. Throughout, singletons are assumed convex.

2.9A. Theorem. Let $X$ be a compact uniform $\mathbf{S}_{4}$ convex structure with connected convex sets. Then all convex sets are acyclic and $X$ has the fixed point property for USC multifunctions $X \rightarrow H(X)$ with acyclic values.

2.9B. Theorem. Let $X$ be a metric $\mathbf{S}_{4}$ convex structure with connected convex sets and with compact polytopes. If $F: Y \rightarrow H(X)$ is a LSC multifunction on a normal space $Y$, such that $F(y)$ is compact and convex for each $y \in Y$, then $F$ has a continuous selection.

2.9C. Theorem. Let $X$ be a metric $\mathbf{S}_{4}$ convex structure with connected convex sets and with compact polytopes, let $Y$ be a metric space, and let $F: Y \rightarrow H(X)$ be a continuous multifunction with compact convex values. If $Y$ has no isolated points and if $Y_{0} \subseteq Y$ is closed and nowhere dense, then for each $\varepsilon>0$ and for each map $f_{0}: Y_{0} \rightarrow X$ with $f(y) \in F(y)$ for all $y \in Y_{0}$, there exists a continuous extension $f: Y \rightarrow X$ of $f_{0}$ such that:

(1) $f$ is a selection of $F$.

(2) The graph of $f$ is $\varepsilon$-close to the graph of $F$.

In the last statement, $Y \times X$ is equipped with the Cartesian metric, for which the distance between two points is the maximum of the coordinate distances.

\section{General spaces of arcs}

An arbitrary Lawson semilattice $S$ may have more than one minimal element. As the set of all minima need not be closed, it is natural then to consider the compact space $\Gamma(S)$ of all arcs in $S$.

3.1. Proposition. Let $S$ be a Lawson semilattice. Then there is a continuous function $e: \Gamma(S) \rightarrow S$, sending an arc to its minimum.

Proof. Let $a=e(A)$ and let $N \subseteq S$ be a neighborhood of $a$. According to [G\&, II.1.10, III.1.5], there exist points $u, \nu_{1}, \ldots, \nu_{n} \in S$ such that the set

$$
U=\left\{x \in S \mid x \leq u ; \forall i: x \not \nu_{i}\right\}
$$

is a neighborhood of $a$ included in $N$. Consider the following set.

$$
\mathscr{U}=\left(\bigcap_{i=1}^{n}\left\langle S \backslash \leq\left(\nu_{i}\right)\right\rangle \cap\langle U, S\rangle \cap \Gamma(S) .\right.
$$

Clearly, $\mathscr{U}$ is a neighborhood of $A$ and each $\operatorname{arc} B \in \mathscr{U}$ must end in $U$.

We will henceforth refer to $e$ as the endpoint map. 
3.2. Convex sets in general spaces of arcs. Throughout, $S$ denotes a general Lawson semilattice. There seems to be no reasonable way to define a "global" convexity on the space $\Gamma(S)$ of all arcs. The following is an attempt to define at least a system of convex sets. A collection of arcs in $\Gamma(S)$ joining the same pair of points in $S$ is henceforth referred to as "admissible". For finite admissible sets, the concept of betweenness survives by considering the common pair of endpoints as the top and bottom element of an order-convex subsemilattice. We say that a set $C \subseteq \Gamma(S)$ is convex provided it is admissible and contains any arc which is in between a finite collection of elements of $C$. The collection of all convex sets does not contain the entire space $\Gamma(S)$; all other requirements of a convex structure are fulfilled. In [V2], the resulting structure is called a convex system.

For Lawson semilattices with a continuous interval operator

$$
(x, y) \mapsto[x, y] \quad(x \leq y)
$$

there is a procedure to extend the convex system of $\Gamma(S)$ to a true convex structure of type $\Lambda(T)$, where $T$ is a Lawson semilattice with a top and bottom element. The following construction is needed.

3.3. A cone construction. Let $X$ be a convex structure. The cone over $X$ is the convex structure $\Delta(X)$ defined as follows. The underlying set is the quotient set

$$
X \times[0,1] \backslash X \times\{1\} .
$$

The equivalence class of $(x, t)$ is denoted by $\langle x, t\rangle$. Note that the apex, $\langle x, 1\rangle$, can be represented with any $x \in X$. We say that $\langle x, t\rangle$ is in between the points $\left\langle x_{1}, t_{1}\right\rangle, \ldots,\left\langle x_{n}, t_{n}\right\rangle$ provided

$$
x \in \operatorname{co}\left\{x_{i} \mid t_{i} \leq t\right\} \text { and } \min _{i} t_{i} \leq t \leq \max _{i} t_{i} .
$$

This yields a convexity by the "usual" remarks on betweenness. Figure 1 exhibits a typical polytope.

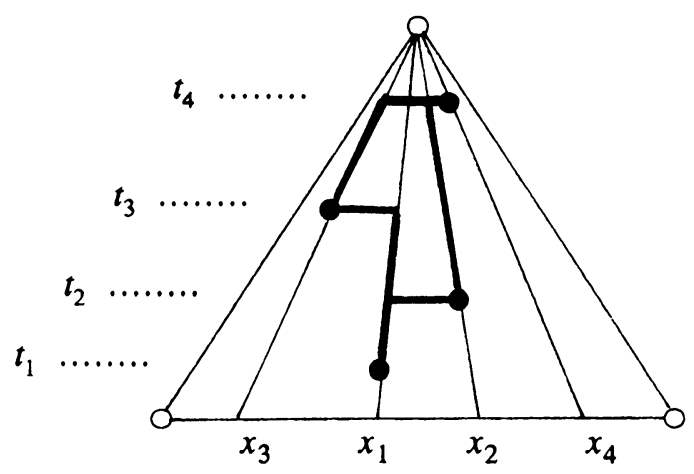

FIGURE 1. Cone convexity 
We note that there is a simpler and seemingly more natural convexity on $\Delta(X)$, consisting of all subcones with a convex basis and restricted between two levels. However, this convexity does not satisfy parts (1) and (2) of the result below. If $X$ is a compact space, then $\Delta(X)$ is topologized as a quotient of the product space $X \times[0,1]$.

\subsection{Proposition. Let $X$ be a compact uniform convex structure. Then:}

(i) $\Delta(X)$ is a compact uniform convex structure.

(ii) $\Delta(X)$ has the Kakutani separation property if $X$ has.

(iii) if all convex sets of $X$ are connected, then the same is true in $\Delta(X)$.

Proof. To avoid working with equivalence classes, we rely on a technique of [vMV]. Let $\square(X)=X \times[0,1]$ be equipped with a convexity, defined as in 3.3 , but without using equivalence classes. We will show that $\square(X)$ satisfies the corresponding statements in (1)-(3). Assuming this to be done, we proceed as follows. The quotient function

$$
q: \square(X) \rightarrow \Delta(X)
$$

preserves convexity both ways, that is: it maps convex sets of $\square(X)$ onto convex sets of $\Delta(X)$ and it inverts convex sets of the latter into convex sets of the former. Then the conclusion of (3) for $\Delta(X)$ follows trivially from the corresponding fact for $\square(X)$. In the terminology of [vMV], $\Delta(X)$ is a uniform convexity quotient of $\square(X)$, and by [vMV, 2.2] it inherits the properties (1) and (2) from $\square(X)$.

Statement 1. $\square(X)$ is a compact uniform convex structure. The underlying space is evidently compact. Its canonical uniformity is generated by symmetric entourages of type

$$
U_{r}=\{((x, s),(y, t)|(x, y) \in U ;| s-t \mid<r\},
$$

where $U \in \mu(X)$ and $r>0$. Let $\varepsilon>0$ and $U \in \mu(X)$. Assume that the sets

$$
\left\{\left(x_{i}, s_{i}\right) \mid i=1, \ldots, m\right\} \text { and }\left\{\left(y_{j}, t_{j}\right) \mid j=1, \ldots, n\right\}
$$

are $V_{\varepsilon}$-close, where $V \in \mu(X)$ is chosen such that $V$-close sets have $U$-close hulls. Let $(x, s)$ be in the hull of the first set, and put $F=\left\{i \mid s_{i} \leq s\right\}$. By construction, $x \in \operatorname{co}\left\{x_{i} \mid i \in F\right\}$. Let $G$ be the collection of all $j$ such that $\left(y_{j}, t_{j}\right)$ is $V_{\varepsilon}$-close to some point $\left(x_{i}, s_{i}\right)$ with $i \in F$. The sets $\left\{x_{i} \mid i \in F\right\}$ and $\left\{y_{j} \mid j \in G\right\}$ are $V$-close and hence there is a point $y \in \operatorname{co}\left\{y_{j} \mid j \in G\right\}$ which is $U$-close to $x$. Let $t$ be the maximum of $t_{j}$ for $j \in G$ and of $s$. As the sets $\left\{s_{i} \mid i \in F\right\}$ and $\left\{t_{j} \mid j \in G\right\}$ are $\varepsilon$-close, and as $s_{i} \leq s$ for all $i \in F$, we find that $0 \leq t-s<\varepsilon$. Hence $(y, t)$ is $U_{\varepsilon}$-close to $(x, s)$. Note that the set of all $j$ such that $t_{j} \leq t$ includes $G$. We conclude that

$$
y \in \operatorname{co}\left\{y_{j} \mid t_{j} \leq t\right\}
$$

and hence that

$$
(y, t) \in \operatorname{co}_{\square}\left\{\left(y_{j}, t_{j}\right) \mid j=1, \ldots, n\right\} .
$$


Statement 2. If $X$ has the Kakutani property, then so does $\square(X)$. Consider the following points of $X \times[0,1]$ :

$$
a_{i}=\left(x_{i}, t_{i}\right) \quad(i=1, \ldots, m) \quad \text { and } \quad b_{j}=\left(y_{j}, s_{j}\right) \quad(j=1, \ldots, n)
$$

and suppose that

$$
\operatorname{co}\left\{a_{1}, \ldots, a_{m}\right\} \cap \operatorname{co}\left\{b_{1}, \ldots, b_{n}\right\}=\varnothing .
$$

We assume that $\max _{i} t_{i} \leq \max _{j} s_{j}$. At the maximal $t_{i}$-level, we see that

$$
\operatorname{co}\left\{x_{1}, \ldots, x_{n}\right\} \cap \operatorname{co}\left\{y_{j} \mid s_{j} \leq \max t_{i}\right\}=\varnothing,
$$

whence there is a half-space $H$ of $X$ with

$$
\operatorname{co}\left\{x_{1}, \ldots, x_{n}\right\} \subseteq H, \quad \text { and } \quad \operatorname{co}\left\{y_{j} \mid s_{j} \leq \max t_{i}\right\} \subseteq X \backslash H .
$$

Then $H \times\left[0, \max t_{i}\right]$ is a half-space of $\square(X)$ and, as the $t$-level section of a convex set decreases when $t$ does, we conclude that this half-space separates between the polytopes $\operatorname{co}\left\{a_{1}, \ldots, a_{n}\right\}$ and $\operatorname{co}\left\{b_{1}, \ldots, b_{n}\right\}$. It follows from [J, I.9] that $\square(X)$ has the property $S_{4}$.

We are now able to prove the announced result. By a subspace of a topological convex structure is meant a subset, equipped with the relative topology and the relative convexity.

3.5. Theorem. Let $S$ be a connected Lawson semilattice with a continuous interval operator. Then there is a connected Lawson semilattice $T$ with a bottom element, and a topological embedding $\Gamma(S) \rightarrow \Lambda(T)$, such that each convex subspace of $\Gamma(S)$ is at the same time a convex subspace of $\Lambda(T)$. Moreover, $T$ is metrizable if $S$ is.

Proof. Let $S$ be convexified by the family of all order-convex subsemilattices. As we already mentioned in $\S 2$, this turns $S$ into a uniform convex structure. We let $\operatorname{Conv}(S)$ denote the convex hyperspace of $S$, that is: the subspace of $H(S)$, consisting of all nonempty convex closed sets. By [V2, 3.6], $\operatorname{Conv}(S)$ is a connected Lawson semilattice under the join operation

$$
C_{1} \vee C_{2}=\mathrm{Clco}\left(C_{1} \cup C_{2}\right) \quad\left(C_{1}, C_{2} \in \operatorname{Conv}(S)\right)
$$

We construct an intermediate embedding

$$
I: \Gamma(S) \rightarrow \Gamma(\operatorname{Conv}(S))
$$

as follows. If $A$ is an arc in $S$ with endpoint $e(A)$, then

$$
I(A)=\{[e(A), a] \mid a \in A\} .
$$

Then $I$ is continuous since the endpoint map $e$ is continuous by Proposition 3.1, and since the order-intervals of $S$ depend continuously on their endpoints. Clearly, $I$ is injective, and hence it is an embedding of compact spaces. By virtue of (1), for each admissible collection $\left(A_{i}\right)_{i=1}^{n}$ of arcs in $S$ we have

$$
I\left(\operatorname{co}\left\{A_{1}, \ldots, A_{n}\right\}\right)=\operatorname{co}\left\{I\left(A_{1}\right), \ldots, I\left(A_{n}\right)\right\}
$$




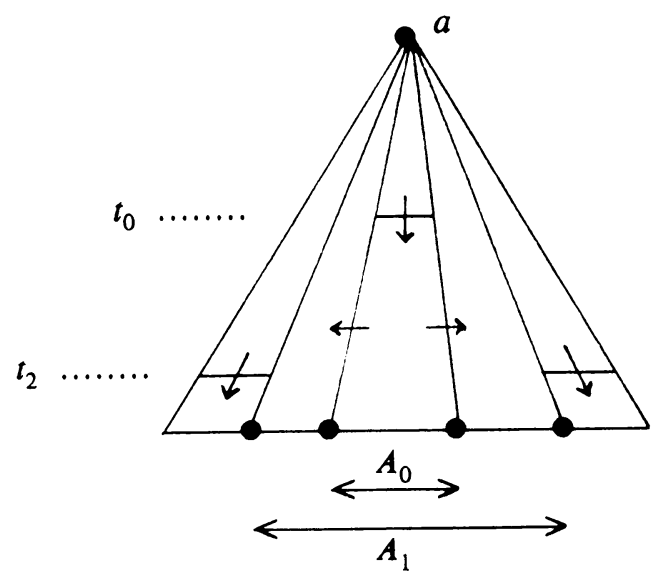

FIGURE 2. The embedding $J$

(first hull in $\Gamma(S)$, second in $\Gamma(\operatorname{Conv}(S)))$. As a consequence, $I$ embeds convex subspaces of $\Gamma(S)$ as convex subspaces of $\Gamma(\operatorname{Conv}(S)))$.

We next consider the cone $\Delta(S)$, which is another uniform $S_{4}$ convex structure by Proposition 3.4. We let $a$ denote its apex. By [V2, 3.6], $\operatorname{Conv}(\Delta(S))$ is a connected Lawson semilattice. The desired semilattice is

$$
T=\operatorname{Conv}_{a}(\Delta(S)),
$$

where the subscript "a" indicates that we only consider sets containing $a$. The bottom element of this new semilattice is $\{a\}$. Moreover, $T$ is connected, and $T$ is metrizable if $S$ is. To complete the proof, we construct a second embedding

$$
J: \Gamma(\operatorname{Conv}(S)) \rightarrow \Lambda(T)
$$

as follows. Let $\mathscr{A} \subseteq \operatorname{Conv}(S)$ be an arc joining the two convex sets $A_{0} \subseteq A_{1}$, and put

$$
J(\mathscr{A})=\mathscr{A}_{0} \cup \mathscr{A}_{1} \cup \mathscr{A}_{2},
$$

where $\mathscr{A}_{0}$ consists of all subcones of type

$$
\left\{\langle x, t\rangle \mid x \in A_{0} ; t \geq t_{0}\right\} \quad\left(t_{0} \in[0,1]\right) .
$$

$\mathscr{A}_{1}$ consists of the cones $\Delta(A)$ over the elements $A \in \mathscr{A}$, and $\mathscr{A}_{2}$ consists of all sets of type

$$
\Delta\left(A_{1}\right) \cup\left\{\langle x, t\rangle \mid x \in S ; t \geq t_{2}\right\} \quad\left(t_{2} \in[0,1]\right) .
$$

See Figure 2. All members of $J(\mathscr{A})$ are convex sets of the cone convexity of $\Delta(S)$, the operation $J$ is easily seen to be continuous (and hence it is a topological embedding), and for each admissible collection $\left(\mathscr{A}_{i}\right)_{i=1}^{n}$ of arcs in $\operatorname{Conv}(S)$ we have

$$
J\left(\operatorname{co}\left\{\mathscr{A}_{1}, \ldots, \mathscr{A}_{n}\right\}\right)=\operatorname{co}\left\{J\left(\mathscr{A}_{1}\right), \ldots, I\left(\mathscr{A}_{n}\right)\right\}
$$

(first hull in $\Gamma(\operatorname{Conv}(S))$, second in $\Lambda(T))$. The desired embedding $\Gamma(S) \rightarrow$ $\Lambda(S)$ is the composed map $J \circ I$. 
A direct consequence of this result is that the theorems quoted in 2.9B and 2.9C also apply to $\Gamma(S)$ with its "system" of convex sets. Note that Theorem 2.9A cannot be transferred this way. In addition to the conclusions of the last theorem, we note that $T=\operatorname{Conv}_{a}(\Delta(S))$ has a continuous interval operator, and is arcwise connected if $S$ is.

\section{Applications}

4.1. Lemma. Let $S, T$ be Lawson semilattices with a bottom element, and let $q: S \rightarrow T$ be a continuous surjective homomorphism. Then the function

$$
\Lambda(q): \Lambda(S) \rightarrow \Lambda(T),
$$

sending an arc of $S$ to its q-image in $T$, is well defined and continuous, and it inverts convex sets of $\Lambda(T)$ into convex sets of $\Lambda(S)$. If all fibers of $q$ are connected, then $\Lambda(q)$ is surjective.

Proof. The assumptions on $q$ imply that $q\left(\mathbf{0}_{S}\right)=\mathbf{0}_{T}$, that $q\left(\mathbf{1}_{s}\right)=\mathbf{1}_{T}$, and that an arc $A \subseteq S$ is transformed into an arc $q(A) \subseteq T$. Hence $\Lambda(q)$ is well defined. Note that this function is a restriction of a function between hyperspaces,

$$
H(S) \rightarrow H(T), \quad A \mapsto q(A),
$$

which is well known to be continuous provided the "basic" function is.

To see that $\Lambda(q)$ inverts convex sets into convex sets, it is sufficient to verify that

$$
\Lambda(q)\left(\operatorname{co}\left\{A_{1}, \ldots, A_{n}\right\}\right) \subseteq \operatorname{co}\left\{\Lambda(q)\left(A_{1}\right), \ldots, \Lambda(q)\left(A_{n}\right)\right\}
$$

for each $A_{1}, \ldots, A_{n} \in \Lambda(S)$. To this end, let the arc $A$ be in between $A_{1}, \ldots, A_{n}$. Then $A \subseteq \bigvee_{i=1}^{n} A_{i}$ and as $q$ is a homomorphism, we find $q(A) \subseteq \mathrm{V}_{i=1}^{n} q\left(A_{i}\right)$, as desired.

Finally, assume that $q$ has connected fibers. It is a fact from general topology that a closed function with connected fibers inverts connected sets to connected sets. Therefore, if $B \in \Lambda(T)$, then the compact subsemilattice $q^{-1}(B)$ of $S$ is connected. It contains the elements $\mathbf{0}_{s}, \mathbf{1}_{s}$, and by Koch's Arc Theorem, there must be an arc $A: \mathbf{0}_{s} \mapsto \mathbf{1}_{s}$ in $q^{-1}(B)$. Evidently, $q(A)=B$.

Along the same lines, it is possible to prove that if all fibers of $q$ are connected, then $\Lambda(q)$ maps convex sets of $\Lambda(S)$ to convex sets of $\Lambda(T)$.

4.2. Invariant Arc Theorem. Let $S, T$ be connected Lawson semilattices with respective bottom elements $\mathbf{0}_{S}, \mathbf{0}_{T}$, let $q: S \rightarrow T$ be a continuous surjective homomorphism with connected fibers, and let $f: S \rightarrow T$ be a continuous function such that if $x \leq y$ in $S$ then $f(x), f(y)$ are comparable in the order of $T$. Suppose that there is an arc in $T$ joining $\mathbf{0}_{T}$ with $f\left(\mathbf{0}_{s}\right)$. Then there exists an $\operatorname{arc} A \in \Lambda(S)$ such that

$$
f(A) \vee f\left(\mathbf{0}_{s}\right) \subseteq q(A) \vee f\left(\mathbf{0}_{s}\right) .
$$

In particular, if $f\left(\mathbf{0}_{S}\right)=\mathbf{0}_{T}$, then $f(A) \subseteq q(A)$ for some $A \in \Lambda(S)$. 
Proof. Consider the mapping

$$
q_{1}: T \rightarrow T_{1}=\left\{t \in T \mid t \geq f\left(\mathbf{0}_{s}\right)\right\}, \quad q_{1}(t)=t \vee f\left(\mathbf{0}_{s}\right) .
$$

Note that $T_{1}$ is a subsemilattice and that $q_{1}$ is a homomorphism. As there exists an $\operatorname{arc} \mathbf{0}_{T} \mapsto f\left(\mathbf{0}_{S}\right)$ in $T$, each fiber of $q_{1}$ is connected. So the composed homomorphism

$$
q_{1} \circ q: S \rightarrow T_{1}
$$

is continuous, surjective, and has connected fibers. We similarly transform $f$ into a mapping

$$
q_{1} \circ f: S \rightarrow T_{1}
$$

preserving comparability of points. These preliminary observations show that it is sufficient to establish the result in case $f$ maps $\mathbf{0}_{S}$ to $\mathbf{0}_{T}$.

Under this assumption, we construct an operator $h: \Lambda(S) \rightarrow \Lambda(T)$ as follows. By Koch's Arc Theorem, there is an arc $A_{0} \subseteq T$ joining $f\left(\mathbf{1}_{s}\right)$ with $\mathbf{1}_{T}$. For $A \in \Lambda(S)$ we define $h(A)$ to be the arc in $T_{1}$ composed of the arcs $f(A)$ and $\sup (f(A)) \vee A_{0}$. This $h$ is easily seen to be well-defined and continuous.

The multivalued function

$$
F: \Lambda(S) \rightarrow \Lambda(S), \quad F(A)=\Lambda(q)^{-1} h(A)
$$

is well-defined since $q$ is surjective, it is upper semicontinuous since $\Lambda(q)$ is a closed mapping, and its value sets are convex by Lemma 4.1. By Theorem 2.9A, the multifunction $F$ has a fixed "point" $A \in \Lambda(S)$. This arc is as desired.

4.3. Comments. (1) It is essential that there be an arc $\mathbf{0}_{T} \mapsto f\left(\mathbf{0}_{s}\right)$ in $T$. For instance let $S=T$ be the (join) subsemilattice of $[0,1]^{2}$, consisting of $[1 / 2,1] \times[0,1]$, together with the line segments joining 0 to $(1 / 3,1)$ and $(1 / 3,1)$ to $(1 / 2,1)$. In addition, let $q$ be the identity homomorphism and consider

$$
f: S \rightarrow S, \quad f(x, y)=((1+x) / 2, y) .
$$

Then $f$ preserves comparability of points (it is even a semilattice homomorphism), but the only arc $A: \mathbf{0} \mapsto \mathbf{1}$ does not satisfy the conclusion of the theorem.

(2) For an order preserving function $f: S \rightarrow S$ on a Lawson semilattice $S$, the following more concrete result can be derived. If $x<y$ are two fixed points of $f$ such that the interval $[x, y]$ is connected, then there exists a point $z$ such that $x<z<y$ and $z, f(z)$ are comparable.

Indeed, $f$ maps $[x, y]$ into itself and preserves the top and bottom elements of this interval. Then take $z$ on some arc $x \mapsto y$ which is mapped into itself by $f$.

It is not difficult to see that this result in turn allows to reprove the Invariant Arc Theorem in case $S=T, q=$ identity, and $f$ is order-preserving.

(3) Let $Y, Z$ be continua and let $f, g: Y \rightarrow Z$ be maps of which $g$ is surjective and has connected fibers. Suppose for a moment that there is a 
collection $\mathscr{C}$ of continua $C \subseteq Y$ with $f(C), g(C)$ incomparable and such that $\mathscr{C}$ separates between the "point" $Y \in C(Y)$ and the collection of singletons of $Y$. Then $f, g$ cannot have a point of coincidence, in other words: $f(y) \neq g(y)$ for all $y \in Y$.

To see this, suppose to the contrary that $f(p)=g(p)=q$, and consider the arcwise connected Lawson semllattices

$$
C_{p}(Y)=\{C \mid p \in C \in C(Y)\} \text { and } C_{q}(Z)=\{C \mid q \in C \in C(Z)\} ;
$$

(the union operator serves as the join). We have induced mappings

$$
C(f), C(g): C_{p}(Y) \rightarrow C_{q}(Z),
$$

where $C(h)(A)=h(A)$ for $h=f, g$. Both are homomorphisms. As all fibers of $g$ are connected, the $g$-preimage of a continuum is a continuum. It follows that $C(g)$ is surjective. By appealing to the Invariant Arc Theorem, we can consider an arc $\mathscr{A}$ of continua joining $\{p\}$ to $Y$, such that $C(f)(\mathscr{A}) \subseteq$ $C(g)(\mathscr{A})$. The arc $\mathscr{A}$ must meet the separating family $\mathscr{C}$, yielding a $C \in \mathscr{C}$ such that $f(C)$ and $g(C)$ are comparable, a contradiction.

For a concrete example of such a family $\mathscr{C}$, one could think of a Whitney level (see below) in $C(Y)$. The above result is somewhat nonintuitive as it actually states that the nonexistence of a coincidence point (a fixed point, if $g=$ identity) can be established by inspecting the behavior of functions "in the large".

4.4. Theorem. Let $S$ be an arcwise connected metric Lawson semilattice with a bottom element, such that the order-intervals $[x, y], x \leq y$, depend continuously on their endpoints. If $X \subseteq S$ is a closed set of mutually incomparable points, separating between $\mathbf{0}$ and $\mathbf{1}$, then $X$ is an absolute retract $(A R)$.

Proof. We construct a function $e_{x}: \lambda(S) \rightarrow X$ as follows. The assumptions on $X$ imply that each arc $A \in \Lambda(S)$ meets $X$ in exactly one point. This point is defined to be $e_{X}(A)$. A general argument in normal topological spaces ensures that such a one-point intersection depends continuously on the intersecting sets.

We next verify that the mapping $e_{X}$ is open. To this end, let $a=e_{X}(A)$ and let $\varepsilon>0$. We consider a sequence of points $\mathbf{0}=a_{0}<a_{1}<\cdots<a_{n+1}=a$, together with a sequence of order-convex open sets $U_{0}, \ldots, U_{n}$ in $S$, such that

(1) $a_{0} \in U_{0} ; a_{n+1} \in U_{n+1} ; \forall i=1, \ldots, n: a_{i} \in U_{i-1} \cap U_{i}$;

(2) $\operatorname{diam}\left(U_{i}\right)<\varepsilon$ for all $i$.

The second property refers to the given metric $d$ of $S$. For $i=0, \ldots, n+1$ we construct a neighborhood $V_{i}$ of $a_{i}$, subject to the following conditions.

(3) $(1 \leq i \leq n), V_{i} \subseteq U_{i-1} \cap U_{i}, V_{0} \subseteq U_{0}, V_{n+1} \subseteq U_{n+1}$.

(4) $(1 \leq i \leq n+1)$, if $x \in V_{i}$ then there is a point $y \in V_{i-1}$ such that $y \leq x$.

This goes by straightforward induction, starting with $V_{0}=U_{0}$ : having obtained $V_{i-1}$, use the fact that $a_{i-1} \in V_{i-1} \cap\left[0, a_{i}\right]$, together with continuity 
of the interval operator to obtain $V_{i}$ as required in (4). Passing to a smaller neighborhood if necessary will yield (3) as well.

Having completed the induction, we finally consider an open subsemilattice $V \subseteq V_{n+1}$ containing $a=a_{n+1}$, and such that

(5) if $s \in S$ and $b \in V$, then $d(s \vee a, s \vee b)<\varepsilon$.

We start with an arbitrary point $b \in V \cap X$. An arc $B$ with $e_{X}(B)=b$ (i.e., $B$ passes through $b$ ) is constructed as follows. Calling upon (4) repeatedly, we obtain a sequence of points

$$
\mathbf{0}=b_{0} \leq \cdots \leq b_{n+1}=b
$$

with $b_{i} \in V_{i}$ for $i=0, \ldots, n+1$. For $0 \leq i \leq n$, fix an arc $B_{i}$ joining $b_{i}$ to $b_{i+1}$. As each $U_{i}$ is order-convex, (3) implies that $B_{i} \subseteq U_{i}$. Hence by (2), the sets $A \cap U_{i}$ and $B_{i}$ are $\varepsilon$-close. It follows that the part of $A$ below $a$ is $\varepsilon$-close to $\bigcup_{i=0}^{n} B_{i}$. On the other hand, $b \vee A$ is an arc joining $b$ to $\mathbf{1}$, and (5) implies that this arc is $\varepsilon$-close to $a \vee A$ (the part of $A$ above $a$ ). Consequently, the arc

$$
B=\bigcup_{i=0}^{n} B_{i} \cup(b \vee A)
$$

is $\varepsilon$-close to $A$.

We have shown that $e_{X}$ maps the $\varepsilon$-ball around $A$ to a subset of $X$ including the $X$-neighborhood $V \cap X$ of $a$. We conclude that $e_{X}$ is open. This fact is used to see that the inverse multivalued function $e_{X}^{-1}: X \rightarrow H(\Lambda(S))$ is lower semicontinuous. The set $e_{X}^{-1}(x)$ consists of all arcs $A \in \Lambda(S)$ passing through the point $x$. This type of set is easily shown to be convex. We are now in a position to apply the Selection Theorem 2.9B, yielding a map $f: X \rightarrow \Lambda(S)$ such that $e_{X} \circ f$ equals the identity of $X$. So $f$ embeds $X$ into $\Lambda(S)$, and $e_{X}$ retracts $\Lambda(S)$ back to $X$. By [V2, Theorem 5.1], a metrizable $S_{4}$ convex structure with connected convex sets and with compact polytopes is an absolute retract. In particular, $\Lambda(S)$ is an AR, and so is its retract $X$.

4.5. Comments. (1) The above result seems to be new even in case of an ordinary cube. In this situation, a simplified argument can be given which avoids the selection theorem to construct a right inverse of $e_{X}$ : for each point $x$ of the separating set $X$ one can consider the arc composed of the line segments $\mathbf{0} x$ and $x \mathbf{1}$. We note, however, that the selection theorem is implicitly needed to show that the space of all $\operatorname{arcs} \mathbf{0} \mapsto \mathbf{1}$ is an AR.

(2) Recall that a Whitney map for a partially ordered space is a map $w: X \rightarrow$ $[0,1]$ with the following properties:

(i) $w(x)=0$ for minimal $x$ and $w(x)=1$ for maximal $x$.

(ii) if $a<b$, then $w(a)<w(b)$.

Ward [W] shows that if $X$ is a compact metrizable pospace in which the sets of maxima and of minima are closed and disjoint, then $X$ has a Whitney map. 
By a Whitney level is meant a set of type $w^{-1}(t)$, where $w$ is a Whitney map and $0<t<1$.

In the circumstances of Theorem 4.4, Ward's result yields a Whitney map on the semilattice $S$, and each Whitney level can take the role of $X$.

(3) For a semilattice $S$ with more than one minimal element, the proof of Theorem 4.4 breaks down. The problem does not lie in the capability to find continuous selections (see the remark following Theorem 3.5) but rather in the following. Let $X$ be a closed set separating between 1 and the set $S_{\min }$ of all minimal elements of $S$. The mapping $e_{X}: \Lambda(S) \rightarrow X$ can be constructed as before, but it need not be open and its fibers need not be admissible (let be convex). These problems could be solved by assuming the existence of a map $g: X \rightarrow S_{\min }$ with $g(x) \leq x$ for all $x \in X$. Instead of selecting from $e_{X}^{-1}$, just take a continuous selection of the convex-valued, continuous function

$$
F: X \rightarrow H(\Lambda(S)), \quad F(x)=\{A \in \Lambda(S) \mid x, g(x) \in A\} .
$$

If $S$ is the hyperspace $H(\mathbf{Z})$ of a continuum $\mathbf{Z}$, then $X$ is a family of closed subsets of $\mathbf{Z}$ and $H(\mathbf{Z})_{\min }$ is the collection of all singletons. In this situation, the function $g$ actually selects a point from each member of $X$. This turns out to be a rather heavy assumption.

On the other hand, the condition that $X$ separates maxima from minima can be relaxed: what is really needed is that any arc joining a minimum with a maximum must pass through $X$. In $H(\mathbf{Z})$, for instance, it suffices to consider subcontinua of $\mathbf{Z}$ as members of $X$. For a metric tree $T$, a selection $g$ on the family of all subcontinua does exist (cf. [N, (5.9); or V2]), and by the previous remarks, it follows that each Whitney level of $C(T)$ is an AR (compare [P], where it is also shown that a Whitney level in $C(\mathbf{Z})$ need not be an AR if $\mathbf{Z}$ is the standard 2-cell). For details on hyperspaces of convex sets, see [V2]. For results involving special Whitney functions, see [GN].

We finally concentrate on Kelley continua. Some definitions have already been given in the introductory section. We find it convenient to develop our results within the following framework. Let $S$ be a connected metric Lawson semilattice. Two points of $S$ are linked provided there is an arc in $S$ joining them; cf. [J2]. We say that $S$ is a Kelley semilattice provided the following condition holds.

(K) For each $\varepsilon>0$ there exists $\delta>0$ such that for all $a, b \in S$ with $d(a, b)<\delta$ and for all $a^{\prime} \geq a$ linked to $a$, there is a point $b^{\prime} \geq b$ which is linked to $b$ and is $\varepsilon$-close to $a^{\prime}$.

4.6. Examples. (1) A compact space is a Kelley continuum iff its hyperspace is a Kelley semilattice. It is evident that $(\mathbf{K})$ is more demanding than the definition of a Kelley continuum. Assume that $Z$ is a Kelley continuum. Let $\varepsilon>0$ and let $\delta>0$ correspond to $\varepsilon$ as required in $(\mathrm{K})$ above. To this end, let $A$, $B \in H(Z)$ and $d(A, B)<\varepsilon$ (the Hausdorff distance is standard). If $A$ is linked to $A^{\prime} \supseteq A$ then $A$ meets each component of $A^{\prime}$. For $a \in A$, let $A^{\prime}(a)$ 
be the component of $a$ in $A^{\prime}$ and let $b \in B$ be $\delta$-close to $a$. The assumptions on $Z$ yield a continuum $B(b, a)$ with the properties

$$
b \in B(b, a) \text { and } d\left(A^{\prime}(a), B(b, a)\right)<\varepsilon .
$$

Note that for each $a \in A$ there is at least one such $b$, and that each $b \in B$ is "involved" with at least one $a \in A$. We put

$$
B^{\prime}=\mathrm{Cl}(\bigcup\{B(b, a) \mid a \in A, b \in B, d(a, b)<\delta\}) .
$$

As $A^{\prime}=\bigcup_{a \in A} A^{\prime}(a)$, we find that $d\left(A^{\prime}, B^{\prime}\right)<\varepsilon$. As $B(b, a)$ is a continuum, Koch's arc theorem gives an arc of continua joining $\{b\}$ to $B(b, a)$. It is not difficult now to produce an arc joining $B$ with $B^{\prime}$.

(2) An arcwise connected Lawson semilattice is a Kelley semilattice. Indeed, let $S$ be as announced. To each $\varepsilon>0$ we associate $\delta>0$ in such a way that $\delta$-close pairs of points have $\varepsilon$-close suprema. If $d(a, b)<\delta$ and $a \leq a^{\prime}$ are linked, then $b^{\prime}=b \vee a^{\prime}$ is as required: the existence of an arc $b \mapsto b^{\prime}$ follows by assumption, whereas

$$
d\left(b^{\prime}, a^{\prime}\right)=d\left(b \vee a^{\prime}, a \vee a^{\prime}\right)<\varepsilon .
$$

(3) A product of two Kelley semilattices is a Kelley semilattice with respect to the coordinatewise join operation and the Cartesian metric. The proof is straightforward.

4.7. Lemma. For a connected metric Lawson semilattice $S$, the following are equivalent.

(1) $S$ is a Kelley semilattice.

(2) The endpoint map $e: \Gamma(S) \rightarrow S$ is open.

In either case, the restricted endpoint map $\Lambda(S) \rightarrow S_{\min }$ is open.

Proof of $(1) \Rightarrow(2)$. Let $A$ be an arc joining $a \leq a^{\prime}$ and let $\varepsilon>0$. Consider a sequence

$$
0<\delta<\delta_{1}<\delta_{2}<\varepsilon
$$

where

(3) $\delta_{2}<\varepsilon$ as in Lemma 1.5 ;

(4) $\delta_{1}<\delta_{2}$ as in Lemma 1.4;

(5) $\delta<\delta_{1}$ as in condition (K) above.

Let $d(a, b)<\delta$ and fix an increasing sequence

$$
a_{0}=a<a_{1}<\cdots<a_{n}=a^{\prime}
$$

( $n=0$ covers the possibility that $a=a^{\prime}$ ), in which successive elements are $\delta$-close. Let $b_{0}^{\prime}=b$. For each $i=1, \ldots, n$ we apply $(\mathbf{K})$ and (5) to obtain a point $b_{i}^{\prime}>b$ which is $\delta_{1}$-close to $a_{i}$, together with an arc $B_{i}^{\prime}$ joining $b$ to $b_{i}^{\prime}$. By (4) and Lemma 1.4 , the $(n+1)$-tuple $\left(a_{0}, \ldots, a_{n}\right)$ is $\delta_{2}$-close to the 
straightening $\left(b_{0}, \ldots, b_{n}\right)$ of $\left(b_{0}^{\prime}, \ldots, b_{n}^{\prime}\right)$. Note: $b_{i}=\bigvee_{j \leq i} b_{j}^{\prime}$. Put $B_{0}=B_{0}^{\prime}$ and, inductively for $1 \leq i<n$,

$$
B_{i+1}=B_{i} \cup\left(b_{i} \vee B_{i+1}^{\prime}\right) .
$$

Note that the expression between brackets is an arc joining $b_{i} \vee b=b_{i}$ to $b_{i} \vee b_{i+1}^{\prime}=b_{i+1}$. We see that $B_{0}$ is a (degenerate) arc $b \mapsto b_{0}=b$. If we know that $B_{i}$ is an arc $b \mapsto b_{i}$, then, apparently, $B_{i+1}$ joins $b$ with $b_{i+1}$ via $b_{i}$. We conclude that $B_{n}$ joins $b$ and $b_{n}$ via $b_{1}, \ldots, b_{n-1}$. By (3) and Lemma 1.5 , the arcs $A$ and $B_{n}$ are $\varepsilon$-close.

Summarizing, we have shown that the endpoint operator $e: \Gamma(S) \rightarrow S_{\min }$ maps the $\varepsilon$-ball at $A \in \Gamma(S)$ to a subset of $S$ including the $\delta$-ball at $e(A) \in S$. Consequently, $e$ is open.

Proof of $(2) \Rightarrow(1)$. By assumption, the multifunction

$$
e^{-1}: S \rightarrow H(\Gamma(S))
$$

is continuous and hence uniformly continuous. Let $\varepsilon>0$, let $\rho>0$ be such that $\rho$-close subsets of $S$ have $\varepsilon$-close suprema, and let $\delta>0$ be such that $\delta$-close points of $S$ have $\rho$-close $e^{-1}$-images. Then $\delta$ is as required in $(\mathrm{K})$.

As for the final part of the lemma, let $A \in \Lambda(S)$ and $a=e(A) \in S_{\min }$. Let $\varepsilon>0$, take $\rho>0$ be such that the order-convex hull of the $\rho$-ball at $\mathbf{1}$ is included in the $\varepsilon$-ball at $\mathbf{1}$, and take $\delta>0$ such that $\delta$-close subsets of $S$ have $\rho$-close suprema. By (2), $e$ maps the $\delta$-ball at $A \in \Gamma(S)$ onto a neighborhood $N$ of $a \in S$. Take $a^{\prime} \in N \cap S_{\text {min }}$ and fix an arc $A^{\prime} \in \Gamma(S)$ which is $\delta$-close to $A$ and with $a^{\prime}=e\left(A^{\prime}\right)$. We see that the points $\sup (A)=1$ and $\sup \left(A^{\prime}\right)$ are $\rho$-close. Consider any arc $A^{\prime \prime}$ joining $\sup \left(A^{\prime}\right)$ with 1 . By assumption, $\operatorname{diam}\left(A^{\prime \prime}\right)<\varepsilon$ and hence the set $A^{\prime} \cup A^{\prime \prime}$ is $\varepsilon$-close to $A$. This yields a member of $\Lambda(S)$ mapping to $a^{\prime}$ under $e$. The result follows easily.

4.8. Lemma. Let $S$ be a Kelley semilattice with a continuous interval operator, such that $S_{\min }$ is compact and has no isolated points. Let $X \subseteq S$ be a closed set of incomparable points, separating between $S_{\min }$ and $\mathbf{1}$, and let $y_{0} \in S_{\min }$ be linked to $x_{0} \in X$; in particular, $y_{0}<x_{0}$. Then for each $\varepsilon>0$ there exists a map $f: S_{\min } \rightarrow X$ with the following properties.

(1) $f\left(y_{0}\right)=x_{0}$.

(2) Each $y \in S_{\min }$ is linked to $f(y)$; in particular, $y<f(y)$.

(3) If $y \in S_{\min }$ is linked to $x \in X$, then there exists a point $a \in S_{\min }$ such that

$$
d(a, y)<\varepsilon \text { and } d(f(a), x)<\varepsilon .
$$

Before proceeding with a proof, let us first translate this result in terms of a hyperspace $H(Z)$, where $Z$ is a Kelley continuum. The interval operator of $H(Z)$ (any compact $Z$ ) is evidently continuous. The subspace $H(Z)_{\min }$ corresponds with $Z$ and hence has no isolated points (at least, if $Z$ is not trivial). For the subspace $X$ of $H(Z)$ we consider a Whitney level $w^{-1}(t)$ 
relative to a Whitney map $w: H(Z) \rightarrow[0,1]$. Note that $a \in Z$ is linked to $A \in H(Z)$ iff $A$ is a continuum and $a \in A$. We arrive at the following formulation.

For each $\varepsilon>0$ there is a continuous multifunction $F: Z \rightarrow C(Z)$ with the following properties.

(i) $\forall z \in Z: w(F(z))=t$ and $z \in F(z)$.

(ii) If $C \subseteq Z$ is a continuum with $w(C)=t$, and if $c \in C$, then there is a point $z \in Z$ with $d(c, z)<\varepsilon, d(C, F(z))<\varepsilon$.

(iii) If $c_{0} \in C_{0}$, where $C_{0} \subseteq Z$ is a continuum with $w\left(C_{0}\right)=t$, then the above multifunction can be chosen such that, moreover, $F\left(c_{0}\right)=C_{0}$.

Proof of the lemma. As in 4.4, we construct a continuous function

$$
e_{X}: \Lambda(S) \rightarrow X
$$

By taking $e_{X}(A)$ equal to the unique point of $A$ in $X$. Let $\varepsilon>0$ and consider $\delta>0$ such that $\delta$-close members of $\Lambda(S)$ map to $\varepsilon$-close points of $X$ under $e_{X}$.

By Lemma 4.7, the endpoint map

$$
e: \Lambda(S) \rightarrow S_{\min }
$$

is open. As $S_{\min }$ is assumed compact, this map is also closed. This leads us to a continuous multifunction

$$
e^{-1}: S_{\min } \rightarrow H(\Lambda(S))
$$

Its value sets

$$
e^{-1}(y)=\{A \in \Lambda(S) \mid y \in A\}
$$

are convex closed. By assumption there is an arc $y_{0} \mapsto x_{0}$, which we compose with an arc $x_{0} \mapsto \mathbf{1}$ (Koch's Arc Theorem). The composed arc $A_{0}$ is in $e^{-1}\left(y_{0}\right)$. We now apply Theorem $2.9 \mathrm{C}$ with respect to $\delta$ and with respect to the preselected $y_{0}, A_{0}$. This yields a continuous selection $g: S_{\min } \rightarrow \Lambda(S)$ of $e^{-1}$ with $g\left(y_{0}\right)=A_{0}$, and such that the graphs of $g$ and of $e^{-1}$ are $\delta$-close. The map

$$
f=e_{X} \circ g: S_{\min } \rightarrow X
$$

satisfies $f\left(y_{0}\right)=x_{0}$ as required in (1). If $y \in S_{\min }$, then $f(y)$ is a point of $X$ on some arc through $y$ as required in (2). Finally, the graphs of $f$ and $e_{X} \circ e^{-1}$ are $\varepsilon$-close. If $y \in S_{\min }$ is linked to $x \in X$, then there is an $\operatorname{arc} A \in e^{-1}(y)$ passing through $x$. We find that $x=e_{X}(A) \subseteq e_{X} e^{-1}(y)$, and by assumption there is a pair $(a, f(a)) \in S_{\min } \times X$ which is $\varepsilon$-close to $(y, x)$. This establishes (3).

A final lemma is needed to eliminate the separating set $X$, appearing in the previous result. 
4.9. Lemma. Let $S$ be a Kelley semilattice with a continuous interval operator, such that $S_{\min }$ is compact and has no isolated points. Let $y_{0} \in S_{\min }$ and let $x_{0} \in S$ be linked with it. Then for each $\varepsilon>0$ there is a map $f: S_{\min } \rightarrow S$ with the following properties.

(i) $f\left(y_{0}\right)=x_{0}$.

(ii) Each element of $S_{\min }$ is linked to its f-image.

(iii) If $y \in S_{\min }$ is linked to $x \in S$ then there is a point $a \in S_{\min }$ with

$$
d(a, y)<\varepsilon \text { and } d(f(a), x)<\varepsilon .
$$

Proof. Fix a Whitney map $w$ of $S$ and consider the following subset of $S \times$ $[0,1]$.

$$
X=\{(s, t) \mid w(s)+t=1\} .
$$

Note that $X$ is the Whitney level of a new Whitney map

$$
S \times[0,1] \rightarrow[0,1], \quad(s, t) \mapsto \frac{1}{2}(w(s)+t),
$$

taken at height $t=1 / 2$. Now $S \times[0,1]$ is another Kelley semilattice inheriting the relevant properties of $S$. Let $p: S \times[0,1] \rightarrow S$ denote the projection to the first coordinate. Then $p$ gives a nonexpansive homeomorphism $X \rightarrow S$ and an isometry $(S \times[0,1])_{\min } \rightarrow S_{\min }$. Applying Lemma 4.8 to $S \times[0,1]$ and projecting to $S$ by $p$ then yields the desired result.

4.10. Theorem. For a metric continuum $Z$, the following assertions are equivalent.

(1) $Z$ is a Kelley continuum.

(2) For each $\varepsilon>0$ and for each $z_{0} \in Z_{0} \in C(Z)$ there is an approximating coselection $F: Z \rightarrow C(Z)$ with $F\left(z_{0}\right)=Z_{0}$.

Proof. We may assume that $Z$ is not a singleton. The hard part of the Theorem, $(1) \Rightarrow(2)$, is a consequence of Lemma 4.9. We just verify that $(2) \Rightarrow(1)$. Consider the multifunction

$$
\mathscr{F}: Z \rightarrow H(C(Z)), \quad z \mapsto\{C \mid z \in C \in C(Z)\} .
$$

Fix any $\varepsilon>0$. An $\varepsilon$-approximating coselection on $Z$ is just a selection of $\mathscr{F}$. As observed by Michael [M, 2.2], a multifunction $\mathscr{F}$ is lower semicontinuous provided for each point $a$ in its domain and for each $b \in \mathscr{F}(a)$, there is a continuous selection $f$ of $\mathscr{F}$ with $f(a)=b$. The lower semicontinuity of the currently considered multifunction expresses exactly the Kelley property of $Z$.

\section{REFERENCES}

[C] D. W. Curtis, Application of a selection theorem to hyperspace contractibility, Canad. J. Math. 37 (1985), 747-759.

[ENN] C. Eberhart, S. B. Nadler, Jr., and W. O. Nowell, Jr., Spaces of order arcs in hyperspaces, Fund. Math. 112 (1981), 111-120. 
[E] R. Engelking, General topology, PWN-Polish Scientific Publishers, Warszawa, 1977.

[G\&] G. Gierz, K. H. Hofmann, K. Keimel, J. D. Lawson, M. Mislove and D. S. Scott, A compendium of continuous lattices, Springer-Verlag, Berlin, 1980, xx+371 pp.

[GN] J. T. Goodykoontz and S. B. Nadler Jr., Whitney levels in hyperspaces of certain Peano continua, Trans. Amer. Math. Soc. 274 (1982), 671-694.

[J1] R. E. Jamison, A general theory of convexity, Dissertation, University of Washington, Seattle, Washington, 1974.

[J2] _ Tietze's convexity theorem for semilattices and lattices, Semigroup Forum 15 (1978), 357-373.

[M] E. Michael, Topologies on spaces of subsets, Trans. Amer. Math. Soc. 71 (1951), 152-182.

[vMV] J. van Mill and M. van de Vel, Equality of the Lebesgue and the inductive dimension functions for compact spaces with a uniform convexity, Colloq. Math. 50 (1986), 187-200.

[N] S. B. Nadler, Hyperspaces of sets, Dekker, New York, 1978, 707 pp.

[P] A. Petrus, Contractibility of Whitney continua in $C(X)$, General Topology Appl. 9 (1978), 275-288.

[V1] M. van de Vel, Pseudo-boundaries and pseudo-interiors for topological convexities, Dissertationes Math. 210 (1983), 1-72.

[V2] _ - A selection theorem for topological convex structures, (to appear).

[V3] _ A Helly property of arcs, Arch. Math. 52 (1989), 298-306.

[W] L. E. Ward Jr., A note on Whitney maps, Canad. Math. Bull. 23 (1980), 373-374.

FakUlteit Wiskunde en INformatika, VRIJe Universiteit, NL-1081 HV, AmSterdam, The NetherLANDS 